\title{
A SPECIAL PARAMETERIZATION OF CURVES
}

\section{BY MARSTON MORSE}

1. Introduction. Parameterization of curves by means of arc length fails when the arc length is infinite. The present paper develops the properties of a special parameterization of curves which never fails to exist and which is most useful in applications. The special parameter is called a $\mu$-length and is an extension of a function of sets defined by $\mathrm{H}$. Whitney* and applied by Whitney to families of simple non-intersecting curves. The curves employed in the present paper are general continuous images of a line segment. This necessitates a slight modification of the definition of Whitney taking order into account. The properties of $\mu$-length developed here are directed largely towards applications in abstract variational theory. While many of Whitney's ideas go over, there are nevertheless certain sharp differences both in the proofs and in the results. In particular the variational theory of general families of closed curves requires concepts not readily suggested either by the functions of Whitney or the extension developed here. The results of the present paper will be applied in the author's paper entitled $A b$ stract variational theory.

2. The $\mu$-Length. Let $N$ be a space of points $p, q, r$ with a metric which is not in general symmetric. That is, to each ordered pair $p, q$ of points of $N$ there shall correspond a number denoted by $p q$ such that $p p=0, p q>0$ if $p \neq q$, and

$$
p q \leqq p r+r q \text {. }
$$

We term $p q$ the distance from $p$ to $q$. Let $|p q|$ denote the maximum of $p q$ and $q p$. We term $|p q|$ the absolute distance between $p$ and $q$. We see that $|p q|=|q p|$ and that $|p q| \leqq|p r|+|r q|$. The points of $N$ taken with the distance $|p q|$ form a metric space which we denote by $|N|$. We shall use the metric of $N$ in defining $\mu$-lengths. For other purposes, in particular in defin-

* H. Whitney, Regular families of curves, Annals of Mathematics, vol. 34 (1933), pp. 244-270. Also Proceedings of the National Academy of Sciences, vol. 18 (1932), pp. 275-278 and pp. 340-342. 
ing continuity on $N$ and $|N|$, we shall use the metric of $|N|$. Let $t$ be a number on a closed interval* $(0, a)$. Let $f(p, t)$ be a single-valued (numerical) function of $p$ and $t$ for $p$ on $|N|$ and $t$ on $(0, a)$. The function $f(p, t)$ will be termed continuous at $(q, \tau)$ if $f(p, t)$ tends to $f(q, \tau)$ as a limit as $|p q|+|t-\tau|$ tends to 0 . For each value of $t$ on $(0, a)$ and point $p$ on $|N|$ let $\phi(p, t)$ be a point on $|N|$. The (point) function $\phi(p, t)$ will be termed continuous at $(q, \tau)$ if the distance $|\phi(p, t) \phi(q, \tau)|$ tends to 0 as a limit as $|p q|+|t-\tau|$ tends to 0 .

Let $p(t)$ be a continuous point function of $t$ for $t$ on $(0, a)$. We term $p=p(t)$ a parameterized curve $\lambda$ (written $p$-curve) and regard two parameterized curves as identical if they are defined by the same point function $p(t)$. We also say that $\lambda$ is the continuous image on $|N|$ of $(0, a)$. In general curves on $|N|$ will be denoted by Greek letters $\alpha, \beta, \cdots$, while points on $|N|$ will be denoted by letters $p, q, r, \cdots$.

Let $\lambda$ be a $p$-curve on $|N|$ given as the continuous image $p=p(t)$ of an interval $0 \leqq t \leqq a$. For $n \geqq 2$ let $t_{1} \leqq t_{2} \leqq \cdots \leqq t_{n}$ be a set of $n$ values of $t$ on $(0, a)$ and let $\left(p_{1}, \cdots, p_{n}\right)=S_{n}$ be the set of corresponding points $p$ on $\lambda$. We term $S_{n}$ an admissible set of $n$ points on $\lambda$. Let the minimum of the numbers $p_{i} p_{i+1}$ as $i$ ranges from 1 to $n-1$ be denoted by $d\left(S_{n}\right)$ and let the least upper bound of all such numbers $d\left(S_{n}\right)$ for a fixed $n$ be denoted by $\mu_{n}(\lambda)$. Following Whitney we then set

$$
\mu_{\lambda}=\frac{\mu_{2}(\lambda)}{2}+\frac{\mu_{3}(\lambda)}{4}+\frac{\mu_{4}(\lambda)}{8}+\cdots .
$$

We term the $\mu_{\lambda}$ the $\mu$-length of $\lambda$.

We enumerate certain properties of $\mu_{n}(\lambda)$ and $\mu_{\lambda}$.

(a) $\mu_{2}(\lambda)=d$, the diameter of $\lambda$.

(b) $\mu_{n}(\lambda) \leqq d$, and $\mu(\lambda) \leqq d$.

(c) $\mu_{n}(\lambda)$ tends to 0 as $n$ becomes infinite.

${ }^{*}$ For the sake of simplicity we assume that $a>0$ and that our $p$-curves do not reduce to points. One could however admit $p$-curves which reduce to points. The $\mu$-lengths of such curves is zero and we would thus admit intervals $(0, a)$ for which $a=0$. For such exceptional curves Fréchet distance is defined in the obvious manner. Theorem 2 is obvious if $\zeta$ reduces to a point. In the proof of Theorem 4 , in case $\lambda_{0}$ reduces to a point, (17) is an easy consequence of $\lambda \lambda_{0}<\delta$ provided $\delta$ is sufficiently small. Otherwise the theorems and proofs hold as written even when the $p$-curves reduce to points. 
(d) $\mu_{n}(\lambda) \geqq \mu_{n+1}(\lambda)$.

(e) If $p(t)$ is not identically constant none of the numbers $\mu_{n}(\lambda)$ is 0 .

Statements (a), (b), (c), and (e) are obvious. To establish (d) let $S_{n+1}$ be any admissible set of $n+1$ points $p_{1}, \cdots, p_{n+1}$ on $\lambda$, $(n>1)$. There is an integer $k$ such that $p_{k} p_{k+1}=d\left(S_{n+1}\right)$. We shall form an admissible set $S_{n}$ on $\lambda$ by removing one point from $S_{n+1}$. If $k \neq 1$ we remove $p_{1}$. If $k=1$ we remove $p_{n+1}$. In both cases the pair $p_{k}, p_{k+1}$ remains and $d\left(S_{n}\right)=d\left(S_{n+1}\right)$. The set of all numbers $d\left(S_{n+1}\right)$ is thus a subset of the numbers $d\left(S_{n}\right)$. Hence (d) is true.

Let $\mu(t)$ be the $\mu$-length of the $p$-curve on $\lambda$ defined by $p=p(\tau)$ for $\tau$ on the interval $(0, t)$. We shall show that $\mu(t)$ has the following properties.

(f) $\mu(t)$ is a continuous, non-decreasing function of $t$.

(g) $\mu(t)$ is constant on each interval on which $p(t)$ is constant.

(h) $\mu(t)$ is constant on no interval on which $p(t)$ is not constant.

It is clear that $\mu(t)$ is non-decreasing. To show that $\mu(t)$ is continuous let $\eta$ be the $p$-curve defined by $p=p(t)$ for $0 \leqq t \leqq \tau$ $<a$, and let $e$ be an arbitrary positive number. There exists a positive number $\delta$ so small that

$$
|p(t) p(\tau)| \leqq \frac{e}{2}, \quad(\tau \leqq t \leqq \tau+\delta \leqq a)
$$

Let $\zeta$ be the $p$-curve defined by $p=p(t)$ for $0 \leqq t \leqq \tau+\delta$. Let $S_{n}^{2}$ be an admissible set of $n$ points on $\zeta$. Let $S_{n}^{1}$ be an admissible set of $n$ points on $\eta$ obtained by replacing each point of $S_{n}^{2}$ for which $t>\tau$ by $p(\tau)$. No point of $S_{n}{ }^{2}$ is thereby moved a distance greater than $e / 2$. Hence

$$
\begin{aligned}
d\left(S_{n}^{2}\right) & \leqq d\left(S_{n}^{1}\right)+e, \\
\mu_{n}(\zeta) & \leqq \mu_{n}(\eta)+e, \\
\mu(\tau+\delta) & \leqq \mu(\tau)+e .
\end{aligned}
$$

Since $\mu(t)$ is non-decreasing, (4) implies continuity on the right. Continuity on the left is similarly established, and the proof of (f) is complete.

Statement $(\mathrm{g})$ requires no proof. To establish (h) we assume that there are values $\tau$ and $\tau^{\prime}$ of $t$ on $(0, a)$ with $\tau^{\prime}>\tau$ such that 
$p(0), p(\tau)$, and $p\left(\tau^{\prime}\right)$ are distinct. Let $h$ and $k$ be the $p$-curves defined by $p(t)$ for $t$ on $(0, \tau)$ and $\left(0, \tau^{\prime}\right)$, respectively. We shall prove that $\mu_{k}>\mu_{h}$. To that end, let $2 c$ be the minimum value of $p(t) p(\tau)+p(t) p\left(\tau^{\prime}\right)$ as $t$ ranges on $(0, \tau)$. We observe that $c \neq 0$ since $p(\tau) \neq p\left(\tau^{\prime}\right)$. Let $S_{n}$ be an admissible set of points $p_{1}, \cdots, p_{n}$ on $h$. We form $S_{n+1}^{\prime}$ on $k$ by adding one point $p_{n+1}$ to $S_{n}$ as follows. If

$$
p_{n} p(\tau) \geqq c,
$$

we add $p(\tau)$. If (5) does not hold we add $p\left(\tau^{\prime}\right)$, and note that

$$
p_{n} p\left(\tau^{\prime}\right) \geqq c
$$

by virtue of our choice of $c$. We suppose $N$ so large an integer that $d\left(S_{n}\right)<c$ for $n>N$. For such values of $n$ it follows from (5) and (6) that $d\left(S_{n+1}^{\prime}\right)=d\left(S_{n}\right)$, and hence

$$
\mu_{n+1}(k) \geqq \mu_{n}(h) .
$$

Since $\mu_{n}(h)$ is not 0 and tends to 0 as $n$ becomes infinite, for some value of $n>N$,

$$
\mu_{n}(h)>\mu_{n+1}(h),
$$

and for such an $n$ it follows from (7) and (8) that $\mu_{n+1}(k)>\mu_{n+1}(h)$. From this relation and from (2) it follows that $\mu_{k}>\mu_{h}$ as stated. The proof of $(\mathrm{h})$ is complete.

3. Equivalent $p$-Curves. Let $\eta$ and $\zeta$ be two $p$-curves given by the respective equations

$$
\begin{array}{ll}
p=p(t), & (0 \leqq t \leqq a), \\
q=q(u), & (0 \leqq u \leqq b) .
\end{array}
$$

Let $H$ be a sense-preserving homeomorphism between the closed intervals $(0, a)$ and $(0, b)$. A homeomorphism of the nature of $H$ will be termed admissible. Let $u=u(t)$ be the value of $u$ corresponding to $t$ under $H$ and let $D(H)$ be the maximum of the distances $|p(t) q[u(t)]|$ as $t$ ranges over $(0, a)$. The Fréchet distance $\eta \zeta$ between $\eta$ and $\zeta$ is the greatest lower bound of the numbers $D(H)$ as $H$ ranges over the set of all admissible homeomorphisms $H$ between $\eta$ and $\zeta$. We observe that $\eta \zeta=\zeta \eta \geqq 0$, and one readily proves that for any three $p$-curves $\eta, \zeta, \lambda, \eta \lambda \leqq \eta \zeta+\zeta \lambda$. 
We shall say that $\eta$ is derivable from $\zeta$ if there exists a continuous non-decreasing function $u=u(t)$ which maps the closed interval $(0, a)$ onto the closed interval $(0, b)$ and such that $p(t) \equiv q[u(t)]$. Two $p$-curves which are derivable from the same $p$-curve $\eta$ will be said to be equivalent.

Let $\mu(t)$ be the $\mu$-length of $\eta$ from the point 0 to the point $t$. Let $t(\mu)$ be the function inverse to $\mu(t)$. To each value of $\mu$ on the interval $0 \leqq \mu=\mu_{\eta}$ there corresponds a value or interval of values $t(\mu)$. We set

$$
p[t(\mu)]=r(\mu), \quad\left(0 \leqq \mu \leqq \mu_{\lambda}\right),
$$

and observe that $r(\mu)$ is single-valued by virtue of $(\mathrm{h}), \S 2$. We shall prove the following proposition.

(A) The function $r(\mu)$ is continuous in $\mu$.

Let $\mu_{0}$ be a value of $\mu(t)$. Corresponding to $\mu_{0}$, let $\tau_{1} \leqq t \leqq \tau_{2}$ be the interval of values taken on by $t(\mu)$ at $\mu_{0}\left(\tau_{1}\right.$ may equal $\left.\tau_{2}\right)$. Corresponding to a positive constant $e$ there exists a positive constant $\delta$ so small that

$$
\left|p\left(\tau_{2}\right) p(t)\right|<e, \quad\left(\tau_{2} \leqq t \leqq \tau_{2}+\delta\right) .
$$

As $t$ ranges over the interval in (12), $\mu(t)$ increases from $\mu_{0}$ to a value $\mu_{1}>\mu_{0}$. From (12) we infer that $\left|r\left(\mu_{0}\right) r(\mu)\right| \leqq e$ for $\mu_{0} \leqq \mu \leqq \mu_{1}$. Thus $r(\mu)$ is continuous on the right at $\mu_{0}$. Continuity on the left is established similarly, and the proof of (A) is complete. We shall now prove the following statement.

(B) The curve $\eta$ is derivable from the curve $r=r(\mu),\left(0 \leqq \mu \leqq \mu_{\eta}\right)$.

It follows from the definition of $r(\mu)$ in (11) that $p(t) \equiv r[\mu(t)]$, and the proof of (B) is complete. We term $r=r(\mu)$ a $\mu$-parameterization of $\eta$ and state the following theorem.

TheOREM 1. Two p-curves are equivalent if and only if they have the same $\mu$-parameterization.

If $\eta$ and $\zeta$ are equivalent, they are derivable from a common $p$-curve $\lambda$ and will have $\mu$-parameterizations identical with that of $\lambda$. Conversely, if $\eta$ and $\zeta$ have a common $\mu$-parameterization $r=r(\mu), \eta$ and $\zeta$ are both derivable from $r=r(\mu)$ in accordance with (B) and hence are equivalent. We shall prove the following theorem. 
THEOREM 2. The Fréchet distance between a p-curve $\zeta$ and $a$ p-curve $\eta$ derivable from $\zeta$ is null.

Suppose $\eta$ and $\zeta$ have the representations (9) and (10), respectively. Suppose $\eta$ is derivable from $\zeta$ under the substitution $u=u(t)$, so that $p(t) \equiv q[u(t)]$ for $t$ on $(0, a)$. Let $c$ be an arbitrarily small positive constant and consider the transformation

$$
u \equiv[u(t)+c t]\left[\frac{b}{b+c a}\right], \quad(0 \leqq t \leqq a) .
$$

This transformation establishes a homeomorphism between the closed intervals $(0, a)$ and $(0, b)$. Denote the right member of (13) by $\phi(t, c)$, and let $\lambda_{c}$ be the $p$-curve

$$
q=q[\phi(t, c)], \quad(0 \leqq t \leqq a) .
$$

The Fréchet distance $\zeta \lambda_{c}=0$. For under the transformation (13) corresponding points of $\zeta$ and $\lambda_{c}$ are identical. To show that $\eta \zeta=0$ we make use of the relation $\eta \xi \leqq \eta \lambda_{c}+\lambda_{c} \zeta=\eta \lambda_{c}$. If $c$ is sufficiently small, points on $\eta$ and $\lambda_{c}$ determined by the same values of $t$ on $(0, a)$ are arbitrarily and uniformly near since $\phi(t, 0) \equiv u(t)$. Hence $\eta \lambda_{c}$ tends to 0 with $c$. But $\eta \zeta$ is independent of $c$ and must then be 0 . The proof of the theorem is complete.

We shall now prove Theorem 3 .

THEOREM 3. If $\eta$ and $\zeta$ are two p-curves for which $\eta \zeta<e$, then $\left|\mu_{\eta}-\mu_{\xi}\right| \leqq 2 e$.

Let $S_{n}$ be an admissible set of $n$ points on $\eta$. There exists an admissible set $S_{n}^{\prime}$ of $n$ points on $\zeta$ with distances from the correspondingly numbered points of $S_{n}$ less than $e$. Hence $\left|d\left(S_{n}^{\prime}\right)-d\left(S_{n}\right)\right| \leqq 2 e$, and we infer that $\left|\mu_{n}(\zeta)-\mu_{n}(\eta)\right| \leqq 2 e$. Upon referring to the definition (2) of $\mu$-length we conclude that Theorem 3 holds as stated. We state the following corollary.

Corollary 1. If $\eta \zeta=0, \mu_{\eta}=\mu_{\zeta}$.

4. Curves. A class of equivalent p-curves will be called a curve class or a curve.

Let $\alpha$ and $\beta$ be two curves. Let $\eta$ and $\eta^{\prime}$ be $p$-curves in the class $\alpha$, and $\zeta$ and $\zeta^{\prime} p$-curves in the class $\beta$. I say that

$$
\eta \zeta=\eta^{\prime} \zeta^{\prime} .
$$


Relation (15) follows from the relation

$$
\eta \zeta \leqq \eta \eta^{\prime}+\eta^{\prime} \zeta^{\prime}+\zeta^{\prime} \zeta .
$$

For $\eta \eta^{\prime}=0$, since $\eta$ and $\eta^{\prime}$ are at a distance 0 from their common $\mu$-parameterized curve $\lambda$ in accordance with Theorem 2. Similarly $\zeta \zeta^{\prime}=0$. Upon reversing the roles of $\eta \zeta$ and $\eta^{\prime} \zeta^{\prime}$ we infer that (15) holds as stated. We are accordingly led to the following statement and definition.

The distance between any two p-curve classes $\alpha$ and $\beta$ equals the distance between any other two p-curves in the classes $\alpha$ and $\beta$, respectively, and will be taken as the distance $\alpha \beta$ between the curves $\alpha$ and $\beta$.

Let $\lambda$ be an arbitrary curve with $\mu$-length $\mu_{\lambda}$. A pair $(\lambda, \mu)$ will be termed admissible if $0 \leqq \mu \leqq \mu_{\lambda}$. For admissible pairs $(\lambda, \mu)$ let $q(\lambda, \mu)$ be the point on $\lambda$ which determines the $\mu$-length $\mu$ on $\lambda$. The following theorem is fundamental.

TheOREM 4. The point function $q(\lambda, \mu)$ is continuous* in its arguments on the domain of admissible pairs $(\lambda, \mu)$.

We shall prove $(\lambda, \mu)$ continuous at $\left(\lambda_{0}, \mu_{0}\right)$ understanding that $\left(\lambda_{0}, \mu_{0}\right)$ is admissible. Let $e$ be an arbitrary positive constant. We shall show that there exists a positive constant $\delta$ such that if $(\lambda, \mu)$ is admissible and

$$
\lambda \lambda_{0}<\delta, \quad\left|\mu-\mu_{0}\right|<\delta,
$$

then

$$
\left|q(\lambda, \mu) q\left(\lambda_{0}, \mu_{0}\right)\right|<e .
$$

To that end we shall subject $\delta$ to two conditions as follows:

(i) We take $\delta<e / 2$. If $\lambda \lambda_{0}<\delta$, there will exist a homeomorphism $T_{\delta}$ between $\mu$-parameterizations of $\lambda$ and $\lambda_{0}$ in which corresponding points have distances less than $\delta$. If the point $\mu$ on $\lambda$ thereby corresponds to $\mu_{1}$ on $\lambda_{0}$,

$$
\left|q(\lambda, \mu) q\left(\lambda_{0}, \mu_{1}\right)\right|<\frac{e}{2} .
$$

* We have not yet shown that for two curves $\alpha$ and $\beta, \alpha \beta=0$ only if $\alpha=\beta$. But this is not necessary to speak of continuity. The proof of our theorem will imply that $q(\alpha, \mu)=q(\beta, \mu)$ when $\alpha \beta=0$. 
(ii) The second condition on $\delta$ is that $\delta$ be so small that when $\left|\mu_{1}-\mu_{0}\right|<3 \delta$,

$$
\left|q\left(\lambda_{0}, \mu_{1}\right) q\left(\lambda_{0}, \mu_{0}\right)\right|<\frac{e}{2} .
$$

This condition can be satisfied by virtue of the continuity of $q\left(\lambda_{0}, \mu\right)$ in $\mu$.

With $\delta$ so chosen I say that (17) holds for admissible pairs $(\lambda, \mu)$ satisfying (16). We introduce $T_{\delta}$ and $\mu_{1}$ on $\lambda_{0}$ as in (i), and recall that

$$
\left|q(\mu, \lambda) q\left(\mu_{0}, \lambda_{0}\right)\right| \leqq\left|q(\lambda, \mu) q\left(\lambda_{0}, \mu_{1}\right)\right|+\left|q\left(\lambda_{0}, \mu_{1}\right) q\left(\lambda_{0}, \mu_{0}\right)\right| .
$$

The first term on the right of (20) is less than $e / 2$ by virtue of (18) and the second term is likewise, provided (19) is applicable; that is, provided $\left|\mu_{1}-\mu_{0}\right|<3 \delta$. But under $T_{\delta}$ a point $\mu$ on $\lambda$ will correspond to a point $\mu_{1}$ on $\lambda_{0}$ such that $\left|\mu-\mu_{1}\right|<2 \delta$ in accordance with Theorem 3. Hence $\left|\mu_{1}-\mu_{0}\right|<3 \delta$, (19) is applicable, and the right member of (20) is less than $e$. The proof of the theorem is complete. We conclude this section with the following theorem.

THEOREM 5. A necessary and sufficient condition that two $p$ curves $\eta$ and $\zeta$ have the same $\mu$-parameterization is that $\eta \zeta=0$.

To prove the condition necessary, let $\lambda$ represent a $\mu$-parameterized curve determined by $\eta$ and $\zeta$. Then $\eta \xi \leqq \eta \lambda+\lambda \zeta$. But $\eta \lambda=\lambda \zeta=0$ by virtue of Theorem 2 . Hence the condition is necessary. To prove the condition sufficient suppose that $\eta \zeta=0$ and let $p=p(\mu),\left(0 \leqq \mu \leqq \mu_{\eta}\right), q=q(\mu),\left(0 \leqq \mu \leqq \mu_{\xi}\right)$, be $\mu$-parameterizations of $\eta$ and $\zeta$, respectively. By virtue of Corollary 1 to Theorem $3, \mu_{\eta}=\mu_{\zeta}$. It then follows from Theorem 4 that $p(\mu)$ and $q(\mu)$ differ by a quantity arbitrarily small in absolute value. Hence $p(\mu)=q(\mu)$, and the condition is proved sufficient. Theorem 5 taken with Theorem 1 gives the following corollary.

Corollary 2. A necessary and sufficient condition that two p-curves $\eta$ and $\zeta$ be equivalent is that $\eta \zeta=0$.

Another way of stating the corollary is to say that for two curves $\alpha$ and $\beta, \alpha \beta=0$ if and only if $\alpha=\beta$.

INSTITUTE FoR AdVANCEd STUdy 